Moroccan J. of Pure and Appl. Anal. (MJPAA)

Volume 4(2), 2018, Pages 158-170

ISSN: Online 2351-8227 - Print 2605-6364

DOI 10.1515/mjpaa-2018-0013

\title{
A few results on some nonlinear parabolic problems in Orlicz-Sobolev spaces
}

\section{Hicham RedwANe $1, a$}

AвstRACT. In this paper, we present our results (see our papers), which concern the existence of the renormalized solutions for equations of the type:

$$
\frac{\partial b(x, u)}{\partial t}-\operatorname{div}(a(x, t, u, \nabla u))-\operatorname{div}(\Phi(x, t, u))=f \quad \text { in } Q=\Omega \times(0, T),
$$

where $b(x, \cdot)$ is a strictly increasing $C^{1}$-function for any $x \in \Omega, a(x, t, s, \xi)$ and $\Phi(x, t, s)$ are a Carathéodory functions. The function $f$ is in $L^{1}(Q)$.

2010 Mathematics Subject Classification. Primary: 35K55, 47A15, 46A32, 47D20.

Key words and phrases. Parabolic equation; Orlicz-Sobolev spaces; Renormalized solutions; Unilateral problem.

\section{Introduction}

Actually, the studies of partial differential equations in modular spaces attract the attention of many researchers motivated by their applications in different domains, many models coming from various branches of mathematical physics, such as elastic mechanics, electro-rheological fluid dynamics and image processing, see for instance $[8,20]$.

In this paper, we survey some existence results for a class of parabolic problems, we refer to [1, 2, 19] for an extended treatment. Let $\Omega$ be a bounded open subset of $\mathbb{R}^{N}, N \geq 2, Q=\Omega \times(0, T)$ where $T$ is a positive real number and $M$ is an Orlicz function. Let $\mathrm{A}(u):=-\operatorname{div}(a(x, t, u, \nabla u))$ be a so-called Leray-Lions type operator whose prototype is the p-Laplacian operator $a(x, s, \xi)=|\xi|^{p-2} \xi$ and $b: \Omega \times \mathbb{R} \rightarrow \mathbb{R}$ is a Carathéodory function such that $b(x, \cdot)$ is a strictly increasing $C^{1}$-function for any fixed $x \in \Omega$ with $b(x, 0)=0$ and $f \in L^{1}(Q)$.

Received February 25, 2019 - Accepted April 15, 2019.

(C) The Author(s) 2017. This article is published with open access by Sidi Mohamed Ben Abdallah University.

${ }^{1}$ Faculté des Sciences Juridiques, Economiques et Sociales. Université Hassan 1er, B.P. 784, Settat, Morocco

a e-mail: redwane_hicham@yahoo.fr . 
Let us consider the following parabolic equation

$$
\frac{\partial b(x, u)}{\partial t}+\mathrm{A}(u)-\operatorname{div}(\Phi(x, t, u))=f \text { in } Q
$$

In the first section, we investigate an existence of renormalized solution for a Cauchy-Dirichlet problem associated to (1.1) in the particular case where the lower order term $\Phi$ is independent of the spacial variable $(x, t)$.

While in the second part, in the case where the Orlicz function $M(t)=t^{p}$, the space framework is then the classical Sobolev space. In this context, we present a study of the unilateral problem associated to equation (1.1), the function $\Phi$ is a nonlinear lower order term satisfying only a growth condition, The aim of this work is to investigate the relationship between the considered obstacle problem and some penalized sequence of approximate equation. We study the possibility of finding a solution of our problem as limit of a subsequence $u_{\epsilon}$ of the

approximate solutions. The penalized term $\frac{1}{\epsilon} T_{\frac{1}{\epsilon}}\left(u_{\epsilon}-\psi\right)^{-}$introduced in the approximate problem plays a crucial role in the proof of such solution.

Finally, the third part is devoted to an existence result for a nonlinear parabolic systems of two equations like (1.1) in the case where $b$ and $\Phi$ depend only on $u$, namely, we take

$$
\frac{\partial b_{i}\left(u_{i}\right)}{\partial t}+\mathrm{A}\left(u_{i}\right)-\operatorname{div}\left(\Phi_{i}\left(u_{i}\right)\right)+f_{i}\left(x, u_{1}, u_{2}\right)=0 \text { in } Q \text { for } i=1,2,
$$

we deal with the renormalized solution for the above system in Sobolev spaces where $f_{i}$ is a Carathéodory function satisfying some growth assumptions.

In what follows, we will use the following real function of a real variable, called the truncation at height $k>0$,

$$
T_{k}(s)=\max (-k, \min (k, s))= \begin{cases}s & \text { if }|s| \leq k \\ k \frac{s}{|s|} & \text { if }|s|>k\end{cases}
$$

\section{EXISTENCE RESULTS FOR A CLASS OF NONLINEAR PARABOLIC EQUATIONS IN ORLICZ SPACES}

2.1. Basic assumptions and main result. Through this paper assume that for any $k>0$, there exists $\lambda_{k}>0$, a function $A_{k} \in L^{\infty}(\Omega)$ and a function $B_{k} \in L_{M}(\Omega)$ such that,

$$
\lambda_{k} \leq \frac{\partial b(x, s)}{\partial s} \leq A_{k}(x) \text { and }\left|\nabla_{x}\left(\frac{\partial b(x, s)}{\partial s}\right)\right| \leq B_{k}(x)
$$

Our main goal in this section is to prove an existence of renormalized solutions in the setting of Orlicz spaces to the following Cauchy-Dirichlet boundary value problem

$$
\begin{cases}\frac{\partial b(x, u)}{\partial t}-\operatorname{div}(a(x, t, u, \nabla u))-\operatorname{div}(\Phi(u))=f & \text { in } Q \\ b(x, u)(t=0)=b\left(x, u_{0}\right) & \text { in } \Omega \\ u=0 & \text { on } \partial \Omega \times(0, T),\end{cases}
$$

where $a: Q \times \mathbb{R} \times \mathbb{R}^{N} \rightarrow \mathbb{R}^{N}$ is a Carathéodory function satisfying, for almost every $(x, t) \in Q$ and for all $s \in \mathbb{R}, \xi, \eta \in \mathbb{R}^{N}(\xi \neq \eta)$ the following conditions

$\left(H_{1}\right)$ : There exists a function $C_{k} \in E_{\bar{M}}(Q)$ and some positive constants $\beta_{k}^{1} \beta_{k}^{2}, \beta_{k}^{3}, \beta_{k}^{4}$ and an Orlicz function $P \prec \prec M$ such that

$$
|a(x, t, s, \xi)| \leq C_{k}(x, t)+\beta_{k}^{1} \bar{M}^{-1}\left(P\left(\beta_{k}^{2}|s|\right)+\beta_{k}^{3} \bar{M}^{-1}\left(M\left(\beta_{k}^{4}|\xi|\right)\right) .\right.
$$

$\left(H_{2}\right)$ : The vector $a$ is strictly monotone

$$
(a(x, t, s, \xi)-a(x, t, s, \eta)) \cdot(\xi-\eta)>0 .
$$

$\left(H_{3}\right): a$ is coercive, there exists a constant $\alpha>0$ such that

$$
a(x, t, s, \xi) \cdot \xi \geq \alpha M(|\xi|) .
$$

$\left(H_{4}\right)$ : For the lower order term, we assume $\Phi: \mathbb{R} \rightarrow \mathbb{R}^{N}$ be a continuous function. 
For that concerns the right hand, $f \in L^{1}(Q)$ and $u_{0} \in L^{1}(\Omega)$,

Lemma 2.1. [19] Under assumptions $\left(H_{1}\right)-\left(H_{3}\right)$, let $\left(Z_{n}\right)$ be a sequence in $W_{0}^{1, x} L_{M}(Q)$ such that

$$
\begin{gathered}
Z_{n} \rightarrow Z \quad \text { in } W_{0}^{1, x} L_{M}(Q) \text { for } \sigma\left(\Pi L_{M}(Q), \Pi E_{\bar{M}}(Q)\right), \\
\left(a\left(x, t, Z_{n}, \nabla Z_{n}\right)\right)_{n} \text { is bounded in }\left(L_{\bar{M}}(Q)\right)^{N}, \\
\lim _{n, s \rightarrow \infty} \int_{Q}\left(a\left(x, t, Z_{n}, \nabla Z_{n}\right)-a\left(x, t, Z_{n}, \nabla Z \chi_{s}\right)\right) \cdot\left(\nabla Z_{n}-\nabla Z \chi_{s}\right) d x d t=0,
\end{gathered}
$$

where $\chi_{s}$ denote the characteristic function of the set $\Omega_{s}=\{x \in \Omega:|\nabla Z| \leq s\}$. Then,

$$
\begin{gathered}
\nabla Z_{n} \rightarrow \nabla Z \text { a.e. in } Q, \\
\lim _{n \rightarrow \infty} \int_{Q} a\left(x, t, Z_{n}, \nabla Z_{n}\right) \nabla Z_{n} d x=\int_{Q} a(x, t, Z, \nabla Z) \nabla Z d x d t, \\
M\left(\left|\nabla Z_{n}\right|\right) \longrightarrow M(|\nabla Z|) \quad \text { in } L^{1}(Q) .
\end{gathered}
$$

Now, we give the definition of a renormalized solution for problem (2.2).

Definition 2.1. A measurable function $u$ defined on $Q$ is said a renormalized solution of problem (2.2) if

$$
\begin{gathered}
T_{k}(u) \in W_{0}^{1, x} L_{M}(Q) \quad \forall k \geq 0, \quad \text { and } \quad b(x, u) \in L^{\infty}\left(0, T, L^{1}(\Omega)\right), \\
\lim _{m \rightarrow \infty} \int_{\{m \leq|u(x, t)| \leq m+1\}} a(x, t, u, \nabla u) \nabla u d x d t=0,
\end{gathered}
$$

and if, for every function $S$ (renormalization) in $W^{2, \infty}(\mathbb{R})$ with compact support, we have

$$
\begin{gathered}
\frac{\partial B_{S}(x, u)}{\partial t}-\operatorname{div}\left(S^{\prime}(u) a(x, t, u, \nabla u)\right)+S^{\prime \prime}(u) a(x, t, u, \nabla u) \nabla u \\
-\operatorname{div}\left(S^{\prime}(u) \Phi(x, t, u)\right)+S^{\prime \prime}(u) \Phi(x, t, u) \nabla u=f S^{\prime}(u) \text { in } \mathcal{D}^{\prime}(Q), \\
B_{S}(x, u)(t=0)=B_{S}\left(x, u_{0}\right) \text { in } \Omega,
\end{gathered}
$$

where $B_{S}(x, \tau)=\int_{0}^{\tau} \frac{\partial b(x, s)}{\partial s} S^{\prime}(s) d s$.

Remark 2.1. [19] For every $S \in W^{2, \infty}(\mathbb{R})$ nondecreasing function such that $\operatorname{supp}\left(S^{\prime}\right) \subset[-k, k]$ and (2.1), we have

$$
\lambda_{k}\left|S\left(s_{1}\right)-S\left(s_{2}\right)\right| \leq\left|B_{S}\left(x, s_{1}\right)-B_{S}\left(x, s_{2}\right)\right| \leq\left\|A_{k}\right\|_{L^{\infty}(\Omega)}\left|S\left(s_{1}\right)-S\left(s_{2}\right)\right|,
$$

for almost every $x \in \Omega$ and for every $s_{1}, s_{2} \in \mathbb{R}$.

The following theorem is our main result.

Theorem 2.1. Suppose that the assumptions $\left(H_{1}\right)-\left(H_{4}\right)$ hold true and $f \in L^{1}(Q)$, then there exists at least a renormalized solution of problem (2.2).

The proof of the above theorem is divided into four steps.

Step 1: Approximate problems. Let $f_{n}$ be a sequence of regular function in $\mathcal{C}_{0}^{\infty}(Q)$ which converges strongly to $f$ in $L^{1}(Q)$ and such that $\left\|f_{n}\right\|_{L^{1}} \leq\|f\|_{L^{1}}$ and for each $n \in \mathbb{N}^{*}$, put

$$
\begin{gathered}
b_{n}(x, s)=T_{n}(b(x, s))+\frac{1}{n} s, \\
a_{n}(x, t, s, \xi)=a\left(x, t, T_{n}(s), \xi\right) \text { a.e }(x, t) \in Q, \forall s \in \mathbb{R}, \forall \xi \in \mathbb{R}^{N},
\end{gathered}
$$

$\Phi_{n}$ is a Lipschitz continuous bounded function from $\mathbb{R}$ into $\mathbb{R}^{N}$, such that $\Phi_{n}$ uniformly converges to $\Phi$ on any compact subset of $\mathbb{R}$ as $n$ tends to $+\infty$. Let $u_{0 n} \in \mathcal{C}_{0}^{\infty}(\Omega)$ such that

$$
\left\|b_{n}\left(x, u_{0 n}\right)\right\|_{L^{1}} \leq\left\|b\left(x, u_{0}\right)\right\|_{L^{1}} \text { and } b_{n}\left(x, u_{0 n}\right) \longrightarrow b\left(x, u_{0}\right) \text { in } L^{1}(\Omega) .
$$


Considering the following approximate problem

$$
\left\{\begin{array}{lr}
\frac{\partial b_{n}\left(x, u_{n}\right)}{\partial t}-\operatorname{div} a\left(x, t, u_{n}, \nabla u_{n}\right)-\operatorname{div} \Phi_{n}\left(u_{n}\right)=f_{n} & \text { in } Q \\
b_{n}\left(x, u_{n}\right)(t=0)=b_{n}\left(x, u_{0}\right) & \text { in } \Omega \\
u_{n}=0 & \text { on } \partial \Omega \times(0, T) .
\end{array}\right.
$$

Thus, from [11], the approximate problem (2.12) has at least one weak solution $u_{n} \in W_{0}^{1, x} L_{M}(Q)$

Step 2: A Priori Estimates.

Proposition 2.1. Suppose that the assumptions $\left(H_{1}\right)-\left(H_{4}\right)$ hold true and let $\left(u_{n}\right)_{n}$ be a solution of the approximate problem (2.12). Then, for all $k>0$, there exists a constant $C$, (not depending on $n$ ), such that:

$$
\begin{gathered}
\left\|T_{k}\left(u_{n}\right)\right\|_{W_{0}^{1, x} L_{M}(Q)} \leq C k \\
\int_{\Omega} B_{k}^{n}\left(x, u_{n}\right)(\sigma) d x \leq k\left(\|f\|_{L^{1}(Q)}+\| b\left(x, u_{0} \|_{L^{1}(\Omega)}\right),\right.
\end{gathered}
$$

for almost any $\sigma \in(0, T)$ where $B_{k}^{n}(x, \tau)=\int_{0}^{\tau} T_{k}(s) \frac{\partial b_{n}(x, s)}{\partial s} d s$, and

$$
\lim _{k \rightarrow+\infty} \lim _{n \rightarrow+\infty} \text { meas }\left\{(x, t) \in Q:\left|u_{n}\right|>k\right\}=0 .
$$

Proof. Testing the approximate problem $(2.12)$ by $T_{k}\left(u_{n}\right) \chi_{(0, \sigma)}$, one has for every $\sigma \in(0, T)$

$$
\begin{aligned}
& \int_{\Omega}\left(B_{k}^{n}\left(x, u_{n}\right)(\sigma)-B_{k}^{n}\left(x, u_{0 n}\right)\right) d x+\int_{Q_{\sigma}} a\left(x, t, u_{n}, \nabla u_{n}\right) \nabla T_{k}\left(u_{n}\right) d x d t \\
& \quad+\int_{Q_{\sigma}} \Phi_{n}\left(u_{n}\right) \nabla T_{k}\left(u_{n}\right) d x d t=\int_{Q_{\sigma}} f_{n} T_{k}\left(u_{n}\right) d x d t .
\end{aligned}
$$

The Lipschitz character of $\Phi_{n}$, Stokes formula together with the boundary condition make it possible to obtain

$$
\int_{Q_{\sigma}} \Phi_{n}\left(u_{n}\right) \nabla T_{k}\left(u_{n}\right) d x d t=0
$$

On the other hand, we have $\left\|f_{n}\right\|_{L^{1}} \leq\|f\|_{L^{1}}$, which implies that

$$
\int_{Q} f_{n} T_{k}\left(u_{n}\right) d x d t \leq k\|f\|_{L^{1}} .
$$

Concerning the first integral in (2.16), we have by construction of $B_{k}^{n}\left(x, u_{n}\right)$,

$$
\int_{\Omega} B_{k}^{n}\left(x, u_{n}\right)(\sigma) d x \geq 0
$$

and

$$
0 \leq \int_{\Omega} B_{k}^{n}\left(x, u_{0 n}\right) d x \leq k \int_{\Omega}\left|b_{n}\left(x, u_{0 n}\right)\right| d x \leq k\left\|b\left(x, u_{0}\right)\right\|_{L^{1}(\Omega)} .
$$

Thanks to $\left(H_{3}\right)$, we deduce

$$
\int_{Q_{\sigma}} M\left(\left|\nabla T_{k}\left(u_{n}\right)\right|\right) d x d t \leq k C .
$$

where $C=\|f\|_{L^{1}(\Omega)}+\left\|b\left(x, u_{0}\right)\right\|_{L^{1}(\Omega)}$. To prove (2.14), we combine (2.16), (2.18), (2.19) and (2.20), (2.21). Finally, we prove (2.15), to this end, since $u_{n} \in W_{0}^{1, x} L_{M}(Q)$ there exists $\lambda>0$ and a constant $C_{0}$ such that

$$
\int_{Q} M\left(\frac{\left|u_{n}\right|}{\lambda}\right) d x d t \leq C_{0}
$$


By using young's inequality, we obtain

$$
\begin{aligned}
\operatorname{meas}\left\{\left|u_{n}\right|>k\right\} \leq & \frac{1}{k} \int_{Q}\left|T_{k}\left(u_{n}\right)\right| d x d t \leq \frac{1}{k} \int_{Q}\left|u_{n}\right| d x d t \\
\leq & \frac{\lambda}{k}\left(\int_{Q} M\left(\frac{\left|u_{n}\right|}{\lambda}\right) d x d t+\int_{Q} \bar{M}(1) d x d t\right) \\
\leq & \frac{\lambda}{k}\left(C_{0}+\bar{M}(1)|Q|\right) \quad \forall n, \quad \forall k>0, \\
& \longrightarrow 0 \quad \text { as } k \longrightarrow \infty .
\end{aligned}
$$

Which implies (2.15).

Proposition 2.2. Let $u_{n}$ be a solution of the approximate problem (2.12), then:

(i) $u_{n} \longrightarrow u$ a.e. in $Q$,

(ii) $b_{n}\left(x, u_{n}\right) \longrightarrow b(x, u)$ a.e. in $Q$,

(iii) $b(x, u) \in L^{\infty}\left(0, T ; L^{1}(\Omega)\right)$.

(iv) $\left\{a\left(x, t, T_{k}\left(u_{n}\right), \nabla T_{k}\left(u_{n}\right)\right)\right\}_{n}$ is bounded in $\left(L_{\bar{M}}(Q)\right)^{N}$,

(v) $\lim _{m \rightarrow+\infty} \lim _{n \rightarrow+\infty} \int_{\left\{m \leq\left|u_{n}\right| \leq m+1\right\}} a\left(x, t, u_{n}, \nabla u_{n}\right) \nabla u_{n} d x=0$.

Proof. (see [19] for a complete proof)

\section{Step 3: Almost everywhere convergence of the gradients.}

Proposition 2.3. Let $u_{n}$ be a solution of the approximate problem (2.12). Then, for all $k \geq 0$ we have (for a subsequence still denoted by $u_{n}$ ): as $n \rightarrow+\infty$,

(i) $\nabla u_{n} \rightarrow \nabla u$ a.e. in $Q$,

(ii) $a\left(x, t, T_{k}\left(u_{n}\right), \nabla T_{k}\left(u_{n}\right)\right) \rightarrow a\left(x, t, T_{k}(u), \nabla T_{k}(u)\right)$ weakly in $\left(L_{\bar{M}}(Q)\right)^{N}$,

(iii) $\quad M\left(\left|\nabla T_{k}\left(u_{n}\right)\right|\right) \rightarrow M\left(\left|\nabla T_{k}(u)\right|\right)$ strongly in $L^{1}(Q)$.

Proof. (see [19])

Step 4: Passing to the limit. The limit $u$ of the approximate solution $u_{n}$ of (2.12) satisfies the renormalization identity

$$
\lim _{m \rightarrow \infty} \int_{\{m \leq|u| \leq m+1\}} a(x, t, u, \nabla u) \nabla u d x d t=0 .
$$

Now, we pass to the limit. Testing the approximate problem (2.12) by $S^{\prime}\left(u_{n}\right)$ with $r \in W^{1, \infty}(\mathbb{R})$ having a compact support such that for $k>0, \operatorname{supp}(r) \subset[-k, k]$ we get

$$
\begin{aligned}
& \frac{\partial B_{r}^{n}\left(x, u_{n}\right)}{\partial t}-\operatorname{div}\left(S^{\prime}\left(u_{n}\right) a\left(x, t, u_{n}, \nabla u_{n}\right)\right)+S^{\prime}\left(u_{n}\right) a\left(x, t, u_{n}, \nabla u_{n}\right) \nabla u_{n} \\
& -\operatorname{div}\left(S^{\prime}\left(u_{n}\right) \Phi\left(x, t, u_{n}\right)\right)+S^{\prime}\left(u_{n}\right) \Phi\left(x, t, u_{n}\right) \nabla u_{n}=f S^{\prime}\left(u_{n}\right) \text { in } \mathfrak{D}^{\prime}(Q),
\end{aligned}
$$

where $B_{r}^{n}(x, \tau)=\int_{0}^{\tau} \frac{\partial b_{n}(x, s)}{\partial s} S^{\prime}(s) d s$.

Our aim here is to pass to the limit in each term in the previous equality, as $n \rightarrow \infty$ in each term of (2.24) (see [19]) to conclude that $u$ satisfies (2.11). It remains to show that $B_{S}(x, u)$ satisfies the initial condition of (2.12). To do this, recall that, $S^{\prime}$ has a compact support, we have $B_{S}^{n}\left(x, u_{n}\right)$ is bounded in $L^{\infty}(Q)$. Moreover, (2.24) and the above considerations on the behavior of the terms of this equation show that $\frac{\partial B_{S}^{n}\left(x, u_{n}\right)}{\partial t}$ is bounded in $L^{1}(Q)+W^{-1, x} L_{\bar{M}}(Q)$. As a consequence, an Aubin's type Lemma (cf [21, Corollary 4] ) implies that $B_{S}^{n}\left(x, u_{n}\right)$ lies in a compact set of $C^{0}\left([0, T] ; L^{1}(\Omega)\right)$. It follows that, $B_{S}^{n}\left(x, u_{n}\right)(t=0)$ converges to $B_{S}(x, u)(t=0)$ strongly in $L^{1}(\Omega)$. Due to remark 2.1 and the fact that $b_{n}\left(x, u_{0 n}\right) \longrightarrow b\left(x, u_{0}\right)$ in $L^{1}(\Omega)$, we conclude that $B_{S}^{n}\left(x, u_{n}\right)(t=0)=$ $B_{S}^{n}\left(x, u_{0 n}\right)$ converges to $B_{S}(x, u)(t=0)$ strongly in $L^{1}(\Omega)$. Then we conclude that $B_{S}(x, u)(t=0)=B_{S}\left(x, u_{0}\right)$ in $\Omega$. 


\section{NONLINEAR PARABOLIC INEQUALITIES WITH LOWER ORDER TERMS}

3.1. Assumptions and statement of main result. Consider the following convex set

$$
\mathbf{K}_{\psi}=\left\{u \in L^{p}\left(0, T ; W_{0}^{1, p}(\Omega): u \geq \psi \text { a.e. in } Q\right\}\right.
$$

where $\psi: \Omega \rightarrow \overline{\mathbb{R}}$ is a measurable function. On the convex $\mathbf{K}_{\psi}$ we assume that

(C): $\psi \in L^{p}\left(0, T ; W_{0}^{1, p}(\Omega)\right) \cap L^{\infty}(Q), \quad \frac{\partial \psi}{\partial t} \in L^{1}(Q)$ such that $u_{0} \geq \psi$.

In this section, we discuss an existence of entropy solutions in the setting of the classical Sobolev spaces to the following obstacle problem

$$
\begin{cases}u \geq \psi & \text { a.e in } Q \\ \frac{\partial b(x, u)}{\partial t}-\operatorname{div} a(x, t, u, \nabla u)+\operatorname{div} \Phi(x, t, u)=f & \text { in } Q \\ b(x, u)(t=0)=b\left(x, u_{0}\right) & \text { in } \Omega \\ u=0 & \text { on } \partial \Omega \times(0, T) .\end{cases}
$$

where $a: Q \times \mathbb{R} \times \mathbb{R}^{N} \rightarrow \mathbb{R}^{N}$ is a Carathéodory function satisfying, for almost every $(x, t) \in Q$ and for all $s \in \mathbb{R}, \xi, \eta \in \mathbb{R}^{N}(\xi \neq \eta)$ the following conditions

(A1): There exists a function $h \in L^{p^{\prime}}(Q)$ and some positive constant $v$ such that

$$
|a(x, t, s, \xi)| \leq v\left(h(x, t)+|\xi|^{p-1}\right) .
$$

(A2): The vector $a$ is strictly monotone

$$
(a(x, t, s, \xi)-a(x, t, s, \eta)) \cdot(\xi-\eta)>0 .
$$

(A3): $a$ is coercive, there exists a constant $\alpha>0$ such that

$$
a(x, t, s, \xi) \cdot \xi \geq \alpha M(|\xi|) .
$$

(A4): For the lower order term, we assume for all $s \in \mathbb{R}$ and for almost every $x \in \Omega$, there exists $c \in L^{\tau}(Q)$, for $\tau=\frac{N+p}{p-1}$ such that

$$
|\Phi(x, t, s)| \leq c(x, t)|s|^{\gamma}, \text { with } \gamma=\frac{N+2}{N+p}(p-1) .
$$

(A5): For that concerns the right hand,

$$
f \in L^{1}(Q) \text { and } u_{0} \in L^{1}(\Omega) .
$$

The main result of this section is the theorem below.

Theorem 3.1. Assume that (A1)-(A5) hold true. Then there exists at least one solution u such that $b(x, u) \in L^{\infty}\left(0, T ; L^{1}(\Omega)\right)$, $b(x, u)(t=0)=b\left(x, u_{0}\right)$ a.e. in $\Omega$ and for all $\left.\left.t \in\right] 0, T\right]$,

$$
\left\{\begin{array}{l}
T_{k}(u) \in L^{p}\left(0, T ; W_{0}^{1, p}(\Omega)\right), \quad u \geq \psi \text { a.e in } Q, \\
\int_{0}^{T}\left\langle\frac{\partial \varphi}{\partial s}, \int_{0}^{u} \frac{\partial b(x, z)}{\partial s} T_{k}^{\prime}(z-\varphi) d z\right\rangle d s \\
+\int_{\Omega} \int_{0}^{u_{0}} \frac{\partial b(x, s)}{\partial s} T_{k}(s-\varphi(0)) d s d x+\int_{Q} a(x, s, u, \nabla u) \nabla T_{k}(u-\varphi) d x d s \\
\quad-\int_{Q} \Phi(x, s, u) \nabla T_{k}(u-\varphi) d x d s \leq \int_{Q} f T_{k}(u-\varphi) d x d s, \\
\forall \varphi \in \mathbf{k}_{\psi} \cap L^{\infty}(Q) \text { with } \varphi(T)=0 \text { such that } \frac{\partial \varphi}{\partial t} \in L^{p^{\prime}}\left(0, T ; W^{-1, p^{\prime}}(\Omega)\right) .
\end{array}\right.
$$


The proof of the above theorem is divided into five steps.

Step 1: Approximate problems. For each $\epsilon>0$, let $f_{\epsilon}$ be a sequence of functions in $L^{p^{\prime}}(Q)$ which converges strongly to $f$ in $L^{1}(Q)$ and put

$$
\begin{gathered}
a_{\epsilon}(x, t, s, \xi)=a\left(x, t, T_{\frac{1}{\epsilon}}(s), \xi\right) \text { a.e }(x, t) \in Q, \forall s \in \mathbb{R}, \forall \xi \in \mathbb{R}^{N}, \\
\Phi_{\epsilon}(x, t, r)=\Phi\left(x, t, T_{\frac{1}{\epsilon}}(r)\right) \text { a.e }(x, t) \in Q, \forall r \in \mathbb{R},
\end{gathered}
$$

And let $u_{0 \epsilon} \in \mathcal{C}_{0}^{\infty}(\Omega)$ such that

$$
b\left(x, u_{0 \epsilon}\right) \longrightarrow b\left(x, u_{0}\right) \text { strongly in } L^{1}(\Omega) .
$$

Considering the following approximate problem

$$
\left\{\begin{array}{lc}
\frac{\partial b\left(x, u_{\epsilon}\right)}{\partial t}-\operatorname{div} a_{\epsilon}\left(x, t, u_{\varepsilon}, \nabla u_{\epsilon}\right)+\operatorname{div} \Phi_{\epsilon}\left(x, t, u_{\epsilon}\right)-\frac{1}{\epsilon} T_{\frac{1}{\epsilon}}\left(u_{\epsilon}-\psi\right)^{-}=f_{\epsilon} & \text { in } Q \\
b\left(x, u_{\epsilon}\right)(t=0)=b_{\epsilon}\left(x, u_{0}\right) & \text { in } \Omega \\
u_{\epsilon}=0 & \text { on } \partial \Omega \times(0, T) .
\end{array}\right.
$$

Thus, from [17], the approximate problem has at least one weak solution $u_{\epsilon}$.

Step 2: A priori estimates. Let $\tau_{1} \in(0, T)$, and $t$ fixed in $\left(0, \tau_{1}\right)$, for a subsequence still indexed by $\varepsilon$,

$$
\begin{gathered}
\int_{Q_{\tau_{1}}}\left|\nabla T_{k}\left(b\left(x, u_{\epsilon}\right)-b(x, \psi)\right)\right|^{p} d x d s \leq C, \\
u_{\epsilon} \text { converges almost every where to } u \text { in } Q, \\
T_{k}\left(u_{\epsilon}\right) \text { converges weakly to } T_{k}(u) \text { in } L^{p}\left(0, T ; W_{0}^{1, p}(\Omega)\right), \\
b\left(x, u_{\epsilon}\right) \rightarrow b(x, u) \text { weakly in } L^{p}\left(0, T ; W_{0}^{1, p}(\Omega)\right), \\
a\left(x, t, T_{k}\left(u_{\epsilon}\right), \nabla T_{k}\left(u_{\epsilon}\right)\right) \rightarrow \xi_{k} \text { weakly in }\left(L^{p^{\prime}}(Q)\right)^{N},
\end{gathered}
$$

as $\epsilon$ tends to 0 for any $k>0$ and

$$
b(x, u) \in L^{\infty}\left(0, T ; L^{1}(\Omega)\right)
$$

Proof. One can consult [1].

Step 3: Intermediate results. In this step, we give some lemma (for a full proof we refer to [1])

Lemma 3.1. The subsequence of $u_{\epsilon}$ defined in Step 1 satisfies

$$
\lim _{m \rightarrow+\infty} \limsup _{\epsilon \rightarrow 0} \frac{1}{m} \int_{\left\{\left|u_{\epsilon}\right| \leq m\right\}} a\left(x, t, u_{\varepsilon}, \nabla u_{\epsilon}\right) \nabla u_{\epsilon} d x d t=0 .
$$

Lemma 3.2. Let $k \geq 0$ be fixed. Let $S$ be a $C^{\infty} \mathbb{R}$-function such that $S(r)=r$ for $|r| \leq k$, and supp $S^{\prime}$ is compact. Then

$$
\begin{gathered}
\liminf _{\mu \rightarrow+\infty} \lim _{\epsilon \rightarrow 0} \int_{0}^{T}\left\langle\frac{\partial b\left(x, u_{\epsilon}\right)}{\partial t}, S^{\prime}\left(b\left(x, u_{\epsilon}\right)-b(x, \psi)\right)\left(T_{k}\left(b\left(x, u_{\epsilon}\right)-b(x, \psi)\right)\right.\right. \\
\left.\left.\left.-T_{k}(b(x, u)-b(x, \psi))\right)_{\mu}\right)\right\rangle d t \geq 0,
\end{gathered}
$$

where $\langle.,$.$\rangle denotes the duality pairing between L^{1}(\Omega)+W^{-1, p^{\prime}}(\Omega)$ and $L^{\infty}(\Omega) \cap W^{1, p}(\Omega)$.

Lemma 3.3. The subsequence of $u_{\epsilon}$ satisfies for any $k \geq 0$

$$
\limsup _{\epsilon \rightarrow 0} \int_{0}^{T} \int_{0}^{t} \int_{\Omega} a\left(x, t, T_{k}\left(u_{\varepsilon}\right), \nabla T_{k}\left(u_{\epsilon}\right)\right) \nabla T_{k}\left(u_{\epsilon}\right) d x d t \leq \int_{0}^{T} \int_{0}^{t} \int_{\Omega} \xi_{k} \nabla T_{k}(u) d x d t .
$$

Lemma 3.4. The subsequence $u_{\epsilon}$ defined in step 1 satisfies for any $k \geq 0$

$$
\begin{gathered}
\lim _{\epsilon \rightarrow 0} \int_{Q}\left(a\left(x, t, T_{k}\left(u_{\varepsilon}\right), \nabla T_{k}\left(u_{\epsilon}\right)\right)-a\left(x, t, T_{k}\left(u_{\varepsilon}\right), \nabla T_{k}(u)\right)\right) \\
\times\left(\nabla T_{k}\left(u_{\epsilon}\right)-\nabla T_{k}(u)\right) d x d t=0,
\end{gathered}
$$




$$
\begin{gathered}
\xi_{k}=a\left(x, t, T_{k}(u), \nabla T_{k}(u)\right) \text { a.e in } Q, \\
a\left(x, t, T_{k}\left(u_{\varepsilon}\right), \nabla T_{k}\left(u_{\epsilon}\right)\right) \nabla T_{k}\left(u_{\epsilon}\right) \rightarrow a\left(x, t, T_{k}(u), \nabla T_{k}(u)\right) \nabla T_{k}(u) \text { as } \epsilon \rightarrow 0
\end{gathered}
$$

\section{Step 4: Renormalization identity.}

Lemma 3.5. [1] The limit $u$ of the subsequence $u_{\epsilon}$ satisfies for any $k \geq 0$

$$
\lim _{m \rightarrow+\infty} \frac{1}{m} \int_{\{|u| \leq m\}} a(x, t, u, \nabla u) \nabla u d x d t=0 \text { and } u \geq \psi \text { a.e in } \Omega .
$$

Step 5: Passing to the limit. The limit $u$ of the subsequence $u_{\epsilon}$ satisfies . Indeed, for $\varphi \in \mathbf{K}_{\psi} \cap L^{\infty}(Q), \quad \frac{\partial \varphi}{\partial t} \in$ $L^{p^{\prime}}\left(0, T ; W^{-1, p^{\prime}}(\Omega)\right)$ such that $\varphi(T)=0$, testing the approximate problem by $T_{k}\left(u_{\epsilon}-\varphi\right)$ and using integration by parts, we have

$$
\begin{aligned}
& \int_{0}^{t}\left\langle\frac{\partial b\left(x, u_{\epsilon}\right)}{\partial s}, T_{k}\left(u_{\epsilon}-\varphi\right)\right\rangle d s-\int_{Q_{t}} a_{\epsilon}\left(x, t, u_{\varepsilon}, \nabla u_{\epsilon}\right) \nabla T_{k}\left(u_{\epsilon}-\varphi\right) d x d s \\
& -\int_{Q_{t}} \Phi_{\epsilon}\left(x, t, u_{\epsilon}\right) \nabla T_{k}\left(u_{\epsilon}-\varphi\right) d x d s-\frac{1}{\epsilon} \int_{Q_{t}} T_{\frac{1}{\epsilon}}\left(u_{\epsilon}-\psi\right)^{-} T_{k}\left(u_{\epsilon}-\varphi\right) d x d s \\
& \quad=\int_{Q_{t}} f_{\epsilon} T_{k}\left(u_{\epsilon}-\varphi\right) d x d s .
\end{aligned}
$$

We pass to the limit in each terms of the above equality as $\epsilon \rightarrow 0$, it follows that $u$ satisfies and by a classical arguments for the use of Aubin's type lemma, $b(x, u)$ verifies the initial condition.

\section{EXISTENCE OF SOLUTIONS FOR NONLINEAR PARABOLIC SYSTEMS VIA WEAK CONVERGENCE OF TRUNCATIONS}

4.1. Assumptions and problem setting. We prove existence of renormalized solution for the following nonlinear parabolic systems

$$
\begin{gathered}
\left(b_{i}\left(u_{i}\right)\right)_{t}-\operatorname{div}\left(a\left(x, u_{i}, D u_{i}\right)+\Phi_{i}\left(u_{i}\right)\right)+f_{i}\left(x, u_{1}, u_{2}\right)=0 \text { in } Q, \\
u_{i}=0 \text { on } \Gamma:=(0, T) \times \partial \Omega, \\
b_{i}\left(u_{i}\right)(t=0)=b_{i}\left(u_{i, 0}\right) \text { in } \Omega,
\end{gathered}
$$

where $i=1,2$. The vector field $a$ satisfies the assumptions below:

$a: \Omega \times \mathbb{R} \times \mathbb{R}^{N} \rightarrow \mathbb{R}^{N}$ is a Carathéodory function and

There exists $\alpha>0$ such that

$$
a(x, s, \xi) \cdot \xi \geq \alpha|\xi|^{p}
$$

for almost every $x \in \Omega$, for every $s \in \mathbb{R}$, for every $\xi \in \mathbb{R}^{N}$.

For each $k>0$, there exists $\beta_{k}>0$ and a function $C_{k}$ in $L^{p^{\prime}}(\Omega)$ such that

$$
|a(x, s, \xi)| \leq C_{k}(x)+\beta_{k}|\xi|^{p-1}
$$

for almost every $x \in \Omega$, for every s such that $|s| \leq k$, and for every $\xi \in \mathbb{R}^{N}$.

The vector field $a$ is monotone with respect to its third argument,

$$
\left(a(x, s, \xi)-a\left(x, s, \xi^{\prime}\right)\right)\left(\xi-\xi^{\prime}\right) \geq 0,
$$

for any $s \in \mathbb{R}$, for any $\left(\xi, \xi^{\prime}\right) \in \mathbb{R}^{2 N}$ and for almost every $x \in \Omega$.

Moreover, we suppose that for $i=1,2$,

$$
\begin{gathered}
\Phi_{i}: \mathbb{R} \rightarrow \mathbb{R}^{N} \text { is a continuous function, } \\
b_{i}: \mathbb{R} \rightarrow \mathbb{R} \text { is a strictly increasing } C^{1} \text {-function with } b_{i}(0)=0,
\end{gathered}
$$


$f_{i}: \Omega \times \mathbb{R} \times \mathbb{R} \rightarrow \mathbb{R}$ is a Carathéodory function with

$$
f_{1}(x, 0, s)=f_{2}(x, s, 0)=0 \text { a.e. } x \in \Omega, \forall s \in \mathbb{R} .
$$

and for almost every $x \in \Omega$, for every $s_{1}, s_{2} \in \mathbb{R}$,

$$
\operatorname{sign}\left(s_{i}\right) f_{i}\left(x, s_{1}, s_{2}\right) \geq 0
$$

The growth assumptions on $f_{i}$ are as follows: For each $k>0$, there exists $\sigma_{k}>0$ and a function $F_{k}$ in $L^{1}(\Omega)$ such that

$$
\left|f_{1}\left(x, s_{1}, s_{2}\right)\right| \leq F_{k}(x)+\sigma_{k}\left|b_{2}\left(s_{2}\right)\right|
$$

a.e. in $\Omega$, for all $s_{1}$ such that $\left|s_{1}\right| \leq k$, for all $s_{2} \in \mathbb{R}$. For each $k>0$, there exists $\lambda_{k}>0$ and a function $G_{k}$ in $L^{1}(\Omega)$ such that

$$
\left|f_{2}\left(x, s_{1}, s_{2}\right)\right| \leq G_{k}(x)+\lambda_{k}\left|b_{1}\left(s_{1}\right)\right|
$$

for almost every $x \in \Omega$, for every $s_{2}$ such that $\left|s_{2}\right| \leq k$, and for every $s_{1} \in \mathbb{R}$. Finally, we assume the following condition on the initial data $u_{i, 0}$ :

$$
u_{i, 0} \text { is a measurable function such that } b_{i}\left(u_{i, 0}\right) \in L^{1}(\Omega) \text {, for } i=1,2 \text {. }
$$

Definition 4.1. A couple of functions $\left(u_{1}, u_{2}\right)$ defined on $Q$ is called a renormalized solution of (4.1)-(4.3) if for $i=1,2$ the function $u_{i}$ satisfies

$$
T_{k}\left(u_{i}\right) \in L^{p}\left(0, T ; W_{0}^{1, p}(\Omega)\right) \text { and } b_{i}\left(u_{i}\right) \in L^{\infty}\left(0, T ; L^{1}(\Omega)\right),
$$

for any $k \geq 0$.

$$
\int_{\left\{(t, x) \in Q ; n \leq\left|u_{i}(x, t)\right| \leq n+1\right\}} a\left(x, u_{i}, \nabla u_{i}\right) \nabla u_{i} d x d t \rightarrow 0 \quad \text { as } n \rightarrow+\infty,
$$

and if, for every function $S$ in $W^{2, \infty}(\mathbb{R})$ which is piecewise $C^{1}$ and such that $S^{\prime}$ has a compact support, we have

$$
\begin{aligned}
& \frac{\partial b_{i, S}\left(u_{i}\right)}{\partial t}-\operatorname{div}\left(S^{\prime}\left(u_{i}\right) a\left(x, u_{i}, \nabla u_{i}\right)\right)+S^{\prime \prime}\left(u_{i}\right) a\left(x, u_{i}, \nabla u_{i}\right) \nabla u_{i} \\
& -\operatorname{div}\left(S^{\prime}\left(u_{i}\right) \Phi_{i}\left(u_{i}\right)\right)+S^{\prime \prime}\left(u_{i}\right) \Phi_{i}\left(u_{i}\right) \nabla u_{i}+f_{i}\left(x, u_{1}, u_{2}\right) S^{\prime}\left(u_{i}\right)=0 \quad \text { in } \mathcal{D}^{\prime}(Q),
\end{aligned}
$$

and

where $b_{i, S}(r)=\int_{0}^{r} b_{i}^{\prime}(s) S^{\prime}(s) d s$.

$$
b_{i, S}\left(u_{i}\right)(t=0)=b_{i, S}\left(u_{i, 0}\right) \text { in } \Omega \text {, }
$$

The main result of this section reads as the theorem.

Theorem 4.1. Under assumptions (4.4)-(4.13), there exists at least a renormalized solution $\left(u_{1}, u_{2}\right)$ of Problem (4.1)-(4.3).

Proof. Step 1. Let us introduce the following regularization of the data: for $\varepsilon>0$ and $i=1,2$

$$
\begin{gathered}
b_{i, \varepsilon}(s)=b_{i}\left(T_{\frac{1}{\varepsilon}}(s)\right)+\varepsilon s \quad \forall s \in \mathbb{R}, \\
a_{\mathcal{\varepsilon}}(x, s, \xi)=a\left(x, T_{\frac{1}{\varepsilon}}(s), \xi\right) \text { a.e. in } \Omega, \forall s \in \mathbb{R}, \forall \xi \in \mathbb{R}^{N}, \\
\Phi_{i, \varepsilon} \text { is a Lipschitz continuous bounded function from } \mathbb{R} \text { into } \mathbb{R}^{N}
\end{gathered}
$$

such that $\Phi_{i}^{\varepsilon}$ converges uniformly to $\Phi_{i}$ on any compact subset of $\mathbb{R}$ as $\varepsilon$ tends to 0 .

$$
\begin{aligned}
& f_{1}^{\mathcal{E}}\left(x, s_{1}, s_{2}\right)=f_{1}\left(x, T_{\frac{1}{\varepsilon}}\left(s_{1}\right), T_{\frac{1}{\varepsilon}}\left(s_{2}\right)\right) \quad \text { a.e. in } \Omega, \forall s_{1}, s_{2} \in \mathbb{R}, \\
& f_{2}^{\varepsilon}\left(x, s_{1}, s_{2}\right)=f_{2}\left(x, T_{\frac{1}{\varepsilon}}\left(s_{1}\right), T_{\frac{1}{\varepsilon}}\left(s_{2}\right)\right) \quad \text { a.e. in } \Omega, \forall s_{1}, s_{2} \in \mathbb{R}, \\
& u_{i, 0}^{\varepsilon} \in C_{0}^{\infty}(\Omega), b_{i, \varepsilon}\left(u_{i, 0}^{\varepsilon}\right) \rightarrow b_{i}\left(u_{i, 0}\right) \quad \text { in } L^{1}(\Omega) \text { as } \varepsilon \text { tends to } 0 .
\end{aligned}
$$

Let us now consider the regularized problem

$$
\begin{gathered}
\frac{\partial b_{i, \varepsilon}\left(u^{\varepsilon}\right)}{\partial t}-\operatorname{div}\left(a_{\varepsilon}\left(x, u^{\varepsilon}, D u^{\varepsilon}\right)+\Phi_{i, \varepsilon}\left(u^{\varepsilon}\right)\right)+f_{i}^{\varepsilon}\left(x, u_{1}^{\varepsilon}, u_{2}^{\varepsilon}\right)=0 \quad \text { in } Q, \\
u_{i}^{\varepsilon}=0 \quad \text { on }(0, T) \times \partial \Omega,
\end{gathered}
$$




$$
b_{i, \varepsilon}\left(u_{i}^{\varepsilon}\right)(t=0)=b_{i, \varepsilon}\left(u_{i, 0}^{\varepsilon}\right) \quad \text { in } \Omega .
$$

In view of (4.8) and (4.18), for $i=1,2$, we have

$$
b_{i, \varepsilon}^{\prime}(s) \geq \varepsilon, \quad\left|b_{i, \varepsilon}(s)\right| \leq \max _{|s| \leq \frac{1}{\varepsilon}}\left|b_{i}(s)\right|+1 \quad \forall s \in \mathbb{R},
$$

In view of (4.5), (4.11) and (4.12), $a_{\varepsilon}, f_{1}^{\varepsilon}$ and $f_{2}^{\varepsilon}$ satisfy: There exists $C_{\varepsilon} \in L^{p^{\prime}}(\Omega), F_{\varepsilon} \in L^{1}(\Omega), G_{\varepsilon} \in L^{1}(\Omega)$ and $\beta_{\varepsilon}>0, \sigma_{\varepsilon}>0, \lambda_{\varepsilon}>0$, such that

$$
\begin{aligned}
& \left|a_{\varepsilon}(x, s, \xi)\right| \leq C_{\varepsilon}(x)+\beta_{\varepsilon}|\xi|^{p-1} \quad \text { a.e. in } x \in \Omega, \forall s \in \mathbb{R}, \forall \xi \in \mathbb{R}^{N} . \\
& \left|f_{1}^{\varepsilon}\left(x, s_{1}, s_{2}\right)\right| \leq F_{\varepsilon}(x)+\sigma_{\varepsilon} \max _{|s| \leq \frac{1}{\varepsilon}}\left|b_{i}(s)\right| \quad \text { a.e. in } x \in \Omega, \forall s_{1}, s_{2} \in \mathbb{R}, \\
& \left|f_{2}^{\varepsilon}\left(x, s_{1}, s_{2}\right)\right| \leq G_{\varepsilon}(x)+\lambda_{\varepsilon} \max _{|s| \leq \frac{1}{\varepsilon}}\left|b_{i}(s)\right| \quad \text { a.e. in } x \in \Omega, \forall s_{1}, s_{2} \in \mathbb{R} .
\end{aligned}
$$

As a consequence, proving the existence of a weak solution $u_{i}^{\varepsilon} \in L^{p}\left(0, T ; W_{0}^{1, p}(\Omega)\right)$ of (4.24)-(4.26) is an easy task (see e.g. [9]).

Step 2. Using $T_{k}\left(u_{i}^{\varepsilon}\right)$ as a test function in (4.24) leads to

$$
\begin{aligned}
& \int_{\Omega} b_{i, \varepsilon}^{k}\left(u_{i}^{\varepsilon}\right)(t) d x+\int_{0}^{t} \int_{\Omega} a_{\varepsilon}\left(x, u_{i}^{\varepsilon}, \nabla u_{i}^{\varepsilon}\right) \nabla T_{k}\left(u_{i}^{\varepsilon}\right) d x d s \\
& +\int_{0}^{t} \int_{\Omega} \Phi_{i, \varepsilon}\left(u_{i}^{\varepsilon}\right) \nabla T_{k}\left(u_{i}^{\varepsilon}\right) d x d s+\int_{0}^{t} \int_{\Omega} f_{i}^{\varepsilon}\left(x, u_{1}^{\varepsilon}, u_{2}^{\varepsilon}\right) T_{k}\left(u_{i}^{\varepsilon}\right) d x d s \\
& =\int_{\Omega} b_{i, \varepsilon}^{k}\left(u_{i, 0}^{\varepsilon}\right) d x
\end{aligned}
$$

for $i=1,2$, for almost every $t$ in $(0, T)$ where $b_{i, \varepsilon}^{k}(r)=\int_{0}^{r} T_{k}(s) b_{i, \varepsilon}^{\prime}(s) d s$. The Lipschitz character of $\Phi_{i, \varepsilon}$, Stokes formula together with the boundary condition (4.25) allow to obtain obtain

$$
\int_{0}^{t} \int_{\Omega} \Phi_{i, \varepsilon}\left(u_{i}^{\varepsilon}\right) \nabla T_{k}\left(u_{i}^{\varepsilon}\right) d x d s=0,
$$

for almost any $t \in(0, T)$. Now, as $0 \leq b_{i, \varepsilon}^{k}\left(u_{i, 0}^{\varepsilon}\right) \leq k\left|b_{i, \varepsilon}\left(u_{i, 0}^{\varepsilon}\right)\right|$ a.e. in $\Omega$, it follows that $0 \leq \int_{\Omega} b_{i, \varepsilon}^{k}\left(u_{i, 0}^{\varepsilon}\right) d x \leq$ $k \int_{\Omega}\left|b_{i, \varepsilon}\left(u_{i, 0}^{\varepsilon}\right)\right| d x$. Since $a_{\varepsilon}$ satisfies (4.19), $f_{i}^{\varepsilon}$ satisfies (4.21), (4.22), we deduce from (4.31) ( taking into account the properties of $b_{i, \varepsilon}^{k}$ and $\left.u_{i, 0}^{\varepsilon}\right)$ that

$$
T_{k}\left(u_{i}^{\varepsilon}\right) \text { is bounded in } L^{p}\left(0, T ; W_{0}^{1, p}(\Omega)\right)
$$

independently of $\varepsilon$ for any $k \geq 0$. Proceeding as in $[4,5]$, we prove that for any $S \in W^{2, \infty}(\mathbb{R})$ such that $S^{\prime}$ is compact (supp $\left.S^{\prime} \subset[-k, k]\right)$

$$
S\left(b_{i, \varepsilon}\left(u_{i}^{\varepsilon}\right)\right) \text { is bounded in } L^{p}\left(0, T ; W_{0}^{1, p}(\Omega)\right),
$$

and

$$
\frac{\partial S\left(b_{i, \varepsilon}\left(u_{i}^{\varepsilon}\right)\right)}{\partial t} \text { is bounded in } L^{1}(Q)+L^{p^{\prime}}\left(0, T ; W^{-1, p^{\prime}}(\Omega)\right),
$$

independently of $\varepsilon$, as soon as $\varepsilon<\frac{1}{k}$.

Step 3. Arguing as in [4,5], estimates (4.30) and (4.31) imply that for a subsequence still indexed by $\varepsilon$, $u_{i}^{\varepsilon}$ converges almost every where to $u_{i}$ in $Q$

and thanks to (4.29),

$$
\begin{gathered}
T_{k}\left(u_{i}^{\varepsilon}\right) \text { converges weakly to } T_{k}\left(u_{i}\right) \text { in } L^{p}\left(0, T ; W_{0}^{1, p}(\Omega)\right), \\
\theta_{n}\left(u_{i}^{\varepsilon}\right) \rightarrow \theta_{n}\left(u_{i}\right) \text { weakly in } L^{p}\left(0, T ; W_{0}^{1, p}(\Omega)\right) \\
a_{\varepsilon}\left(x, T_{k}\left(u_{i}^{\varepsilon}\right), \nabla T_{k}\left(u_{i}^{\varepsilon}\right)\right) \rightarrow X_{i, k} \text { weakly in }\left(L^{p^{\prime}}(Q)\right)^{N} .
\end{gathered}
$$


as $\varepsilon$ tends to 0 for any $k>0$ and any $n \geq 1$. Here, for any $k>0$ and for $i=1,2, X_{i, k}$ belongs to $\left(L^{p^{\prime}}(Q)\right)^{N}$. We now establish that $b_{i}\left(u_{i}\right)$ belongs to $L^{\infty}\left(0, T ; L^{1}(\Omega)\right)$. Indeed using $\frac{1}{\sigma} T_{\sigma}\left(u_{i}^{\varepsilon}\right)$ as a test function in (4.24) leads to

$$
\begin{aligned}
& \frac{1}{\sigma} \int_{\Omega} b_{i, \varepsilon}^{\sigma}\left(u_{i}^{\varepsilon}\right)(t) d x+\frac{1}{\sigma} \int_{0}^{t} \int_{\Omega} a_{\varepsilon}\left(x, u_{i}^{\varepsilon}, \nabla u_{i}^{\varepsilon}\right) \nabla T_{\sigma}\left(u_{i}^{\varepsilon}\right) d x d s \\
& +\frac{1}{\sigma} \int_{0}^{t} \int_{\Omega} \Phi_{i, \varepsilon}\left(u_{i}^{\varepsilon}\right) \nabla T_{\sigma}\left(u_{i}^{\varepsilon}\right) d x d s+\frac{1}{\sigma} \int_{0}^{t} \int_{\Omega} f_{i}^{\varepsilon}\left(x, u_{1}^{\varepsilon}, u_{2}^{\varepsilon}\right) T_{\sigma}\left(u_{i}^{\varepsilon}\right) d x d s \\
& =\frac{1}{\sigma} \int_{\Omega} b_{i, \varepsilon}^{\sigma}\left(u_{i, 0}^{\varepsilon}\right) d x,
\end{aligned}
$$

for almost any $t$ in $(0, T)$. Where, $b_{i, \varepsilon}^{n}(r)=\int_{0}^{r} b_{i, \varepsilon}^{\prime}(s) T_{\sigma}(s) d s$. The Lipschitz character of $\Phi_{\varepsilon}$, Stokes formula together with the boundary condition (4.25) allow to obtain

$$
\frac{1}{\sigma} \int_{0}^{t} \int_{\Omega} \Phi_{i, \varepsilon}\left(u_{i}^{\varepsilon}\right) \nabla T_{\sigma}\left(u_{i}^{\varepsilon}\right) d x d s=0 .
$$

Since $a_{\varepsilon}$ satisfies (4.4) and $f_{i}^{\varepsilon}$ satisfies (4.10), letting $\sigma$ go to zero, it follows that

$$
\int_{\Omega}\left|b_{i, \varepsilon}\left(u_{i}^{\varepsilon}\right)(t)\right| d x \leq\left\|b_{i, \varepsilon}\left(u_{i, 0}^{\varepsilon}\right)\right\|_{L^{1}(\Omega)}
$$

for almost $t \in(0, T)$. Recalling (4.23), (4.32) and (4.38) makes it possible to pass to the limit-inf and we show that $b_{i}\left(u_{i}\right)$ belongs to $L^{\infty}\left(0, T ; L^{1}(\Omega)\right)$. The pointwise convergence of $u^{\varepsilon}$ to $u$ and $b_{i, \varepsilon}\left(u_{0}^{\varepsilon}\right)$ to $b_{i}\left(u_{0}\right)$ imply that

$$
\limsup _{\varepsilon \rightarrow 0} \int_{0}^{t} \int_{\Omega} a\left(x, u_{i}^{\varepsilon}, D u_{i}^{\varepsilon}\right) D \theta_{n}\left(u_{i}^{\varepsilon}\right) d x d s \leq \int_{\Omega} b_{i}^{n}\left(u_{i, 0}\right) d x,
$$

Since $\theta_{n}$ converge to zero everywhere as $n$ goes to zero, the Lebesgue's convergence theorem permits to conclude that

$$
\lim _{n \rightarrow+\infty} \limsup _{\varepsilon \rightarrow 0} \int_{\left\{n \leq\left|u_{i}^{\varepsilon}\right| \leq n+1\right\}} a_{\varepsilon}\left(x, u_{i}^{\varepsilon}, \nabla u_{i}^{\varepsilon}\right) \nabla u_{i}^{\varepsilon} d x d t=0 .
$$

Step 4. In this step we give the following Lemma which is the key point in the monotonicity arguments.

Lemma 4.1. The subsequence of $u^{\varepsilon}$ defined in Step 3 satisfies: For any $k \geq 0$,

$$
\begin{aligned}
& \limsup _{\varepsilon \rightarrow 0} \int_{0}^{T} \int_{0}^{t} \int_{\Omega} a\left(u_{i}^{\varepsilon}, \nabla T_{k}\left(u_{i}^{\varepsilon}\right)\right) \nabla T_{k}\left(u_{i}^{\varepsilon}\right) d x d s d t \\
& \leq \int_{0}^{T} \int_{0}^{t} \int_{\Omega} X_{i, k} \nabla T_{k}\left(u_{i}\right) d x d s d t
\end{aligned}
$$

\section{Step 5. Monotonicity estimate.}

Lemma 4.2. The subsequence of $u_{i}^{\varepsilon}$ defined in step 3 satisfies: For any $k \geq 0$,

$$
\begin{aligned}
& \lim _{\varepsilon \rightarrow 0} \int_{0}^{T} \int_{0}^{t} \int_{\Omega}\left[a\left(T_{k}\left(u_{i}^{\varepsilon}\right), \nabla T_{k}\left(u_{i}^{\varepsilon}\right)\right)-a\left(T_{k}\left(u_{i}^{\varepsilon}\right), \nabla T_{k}\left(u_{i}\right)\right)\right] \\
& \times\left[\nabla T_{k}\left(u_{i}^{\varepsilon}\right)-\nabla T_{k}\left(u_{i}\right)\right] d x d s d t=0 .
\end{aligned}
$$

Step 6. In this step we identify the weak limit $X_{i, k}$ and we prove the weak $L^{1}$ convergence of the "truncated" energy $a\left(T_{k}\left(x, u_{i}^{\varepsilon}\right), \nabla T_{k}\left(u_{i}^{\varepsilon}\right)\right) \nabla T_{k}\left(u_{i}^{\varepsilon}\right)$ as $\varepsilon$ tends to 0 .

Lemma 4.3. For fixed $k \geq 0$, as $\varepsilon$ tends to 0 , we have

$$
X_{i, k}=a\left(x, T_{k}\left(u_{i}^{\varepsilon}\right), \nabla T_{k}\left(u_{i}^{\varepsilon}\right)\right) \text { a.e. in } Q \text {. }
$$

Also, as $\varepsilon$ tends to 0 ,

$$
a\left(T_{k}\left(u_{i}^{\varepsilon}\right), \nabla T_{k}\left(u_{i}^{\varepsilon}\right)\right) \nabla T_{k}\left(u_{i}^{\varepsilon}\right) \rightarrow a\left(T_{k}\left(u_{i}\right), \nabla T_{k}\left(u_{i}\right)\right) \nabla T_{k}\left(u_{i}\right),
$$

weakly in $L^{1}(Q)$. 
Step 7. In this step, $u_{i}$ is shown to satisfy (4.16) and (4.17). Let $S$ be a function in $W^{2, \infty}(\mathbb{R})$ such that $S^{\prime}$ has a compact support. Let $k$ be a positive real number such that supp $S^{\prime} \subset[-k, k]$. Pointwise multiplication of the approximate equation $(4.24)$ by $S^{\prime}\left(u_{i}^{\varepsilon}\right)$ leads to

$$
\begin{aligned}
& \frac{\partial b_{i, S}^{\varepsilon}\left(u_{i}^{\varepsilon}\right)}{\partial t}-\operatorname{div}\left(S^{\prime}\left(u_{i}^{\varepsilon}\right) a_{\varepsilon}\left(x, u_{i}^{\varepsilon}, \nabla u_{i}^{\varepsilon}\right)\right)+S^{\prime \prime}\left(u_{i}^{\varepsilon}\right) a_{\varepsilon}\left(x, u_{i}^{\varepsilon}, \nabla u_{i}^{\varepsilon}\right) \nabla u_{i}^{\varepsilon} \\
& -\operatorname{div}\left(S^{\prime}\left(u_{i}^{\varepsilon}\right) \Phi_{i, \varepsilon}\left(u_{i}^{\varepsilon}\right)\right)+S^{\prime \prime}\left(u_{i}^{\varepsilon}\right) \Phi_{\varepsilon}\left(u_{i}^{\varepsilon}\right) \nabla u_{i}^{\varepsilon}+f_{i}^{\varepsilon}\left(x, u_{1}^{\varepsilon}, u_{2}^{\varepsilon}\right) S^{\prime}\left(u_{i}^{\varepsilon}\right)=0
\end{aligned}
$$

in $D^{\prime}(Q)$, for $i=1,2$. In what follows we pass to the limit as $\varepsilon$ tends to 0 in each term of (4.45).

As a consequence of the convergence result of each term above (see [2]), we pass to the limit as $\varepsilon$ tends to 0 in equation (4.45) and we conclude that $u$ satisfies (4.16). It remains to show that $b_{i, S}\left(u_{i}\right)$ satisfies the initial condition (4.17). To this end, firstly remark that, $S$ being bounded, $b_{i, S}^{\varepsilon}\left(u_{i}^{\varepsilon}\right)$ is bounded in $L^{\infty}(Q)$. Secondly, (4.45) and the above considerations on the behavior of the terms of this equation show that $\frac{\partial b_{i, S}^{\varepsilon}\left(u_{i}^{\varepsilon}\right)}{\partial t}$ is bounded in $L^{1}(Q)+L^{p^{\prime}}\left(0, T ; W^{-1, p^{\prime}}(\Omega)\right)$. As a consequence, an Aubin's type lemma (see, e.g, [21], Corollary 4$), b_{i, S}^{\varepsilon}\left(u_{i}^{\varepsilon}\right)$

lies in a compact set of $C^{0}\left([0, T] ; W^{-1, s}(\Omega)\right)$ for any $s<\inf \left(p^{\prime}, \frac{N}{N-1}\right)$. It follows that $b_{i, S}^{\varepsilon}\left(u_{i}^{\varepsilon}\right)(t=0)=b_{i, S}^{\varepsilon}\left(u_{i, 0}^{\varepsilon}\right)$ converges to $b_{i, S}\left(u_{i}\right)(t=0)$ strongly in $W^{-1, S}(\Omega)$. On the order hand, (4.26) and the smoothness of $S$ imply that $b_{i, S}^{\varepsilon}\left(u_{i, 0}^{\varepsilon}\right)$ converges to $b_{i, S}\left(u_{i, 0}\right)(t=0)$ strongly in $L^{q}(\Omega)$ for all $q<+\infty$ and this in turn implies (4.17).

\section{References}

[1] Aberqi, A., Bennouna, J., Mekkour, M., Redwane, H.: Nonlinear parabolic inequalities with lower order terms, Applicable Analysis, DOI: 10.1080/00036811.2016.1205186

[2] Azroul, E., Redwane, H., Rhoudaf, M.: Existence of Solution for Nonlinear Parabolic Systems via Weak Convergence of Truncations, Electronic Journal of Differential Equations, Vol. 2010(2010), No. 68, pp. 1-18. ISSN: 1072-6691. URL: http:/ / ejde.math.txstate.edu

[3] Bennouna, J., Hammoumi, M., Aberqi, A.: Nonlinear degenerated parabolic equations with lower order terms, Electronic Journal of Mathematical Analysis and Applications, pp. 234-253 (2016)

[4] Blanchard, D., Murat, F.: Renormalized Solutions of Nonlinear Parabolic Problems with $L^{1}$ Data, Existence and Uniqueness , Proc. R. Soc. Edinburgh Sect. A 127, 1137-1152 (1997)

[5] Blanchard, D., Murat, F., Redwane, H.: Existence et unicité de la solution renormalisée d'un problème parabolique assez général, C. R. Acad. Sci. Paris Sér. 1329, 575-580 (1999)

[6] Blanchard, D., Porretta, A.: A Stefan Problems with Diffusion and Convection, Differ. Equ. 210, 383-428 (2005)

[7] Boccardo, L., Dall'Aglio, A., Orsina, L.: Existence and regularity results for some elliptic equations with degenerate coercivity. Atti Semin. Mat. Fis. Univ. Modena 46-suppl., 51-81 (1998)

[8] Chen, Y., Levine, S. and Rao, M.: Variable exponent linear growth functonals in image restoration. SIAM J. Appl. Math. 66 (2006) 1383-1406.

[9] Eden, A., Michaux, B., Rakotoson, J.: Doubly Nonlinear Parabolic-Type Equations as Dynamical systems, Journal of Dynamics and Differential Equations, 3, (1991), 87-131.

[10] Elmahi, A., Meskine, D.: Strongly Nonlinear parabolic equations with natural growth terms and $L^{1}$ data in Orlicz spaces, Port. Math. Nova 62, 143-183 (2005)

[11] Elmahi, A., Meskine, D.: Strongly nonlinear parabolic equations with natural growth terms and $L^{1}$ data in Orlicz spaces. Portugaliae Mathematica. Nova 62, 143-183 (2005)

[12] Gossez, J. P.: A strongly nonlinear elliptic problem in Orlicz-Sobolev spaces, Proc. Sympos. Pure Math. 45, Amer. Math. Soc., 455-462 (1986)

[13] Gossez, J. P.: Surjectivity results for pseudo-monotone mappings in complementary systems, J. Math. Anal. Appl. 53, 484-494 (1976)

[14] Gossez, J.P.: Nonlinear elliptic boundary value problems for equations with rapidly (or slowly) increasing coefficients, Trans. Amer. Math. soc. 190, 163-205 (1974)

[15] Gossez, J.P., Mustonen, V.: Variationnal inequalities in Orlicz-Sobolev spaces., Nonlinear Anal. 11, 317-492 (1987) 
[16] Krasnosel'skii, M., Rutikii, Ya.: Convex functions and Orlicz spaces, Groningen, Nordhooff (1969)

[17] Lions, JL.: Quelques méthodes de resolution des probl?mes aux limites non lin?aires [Some methods of resolution of problems with nonlinear boundary]. Paris: Dunod et Gauthier-Villars; 1969.

[18] Musielak, J.: Orlicz spaces and modular spaces, Lecture Note in Mahtematics, 1034, Springer, Berlin, (1983)

[19] Redwane, H.: Existence Results For a Class of Nonlinear Parabolic Equations in Orlicz Spaces, Electronic Journal of Qualitative Theory of Differential Equations, No. 2, 1-19 (2010)

[20] Ruzicka, M.: Electroheological Fluides Modeling and Mathematical Theory. Lecture Notes in Mathematics, 1748. Springer-Verlaag, Berlin, 2000.

[21] Simon, J.: Compact sets in $L^{p}(0, T ; B)$, Ann. Mat. Pura Appl., 146, pp. 65-96 (1987) 